\section{Hypochondriacal Symptoms as the First Sign of Frontotemporal Dementia}

To the Editor: Frontotemporal dementia is a clinicopathological entity characterized by prominent changes in behavior and personality resulting from frontal and temporal lobe degeneration. ${ }^{1}$ Neuropsychiatric features can be so conspicuous as to completely overshadow cognitive dysfunction, usually leading to diagnostic flaw or delay. ${ }^{2}$ Among these, hypochondriacal symptoms can be found, but usually along with other neuropsychiatric core features such as disrupted social and personal conduct, emotional blunting, and striking lack of insight. ${ }^{3}$

\section{Case Report}

A 70-year-old previously healthy woman was seen for progressive behavioral disturbance. Nine years previously she started complaining of having almost all possible diseases. Every time that she saw a documentary or any news article describing a disease, she said she had all its symptoms so she must have the disease. The family started to find this strange because every time she sought medical help and ancillary tests, everything was completely normal. Even stranger was when she feared having completely unusual diseases. On one occasion she thought she had "the blue baby disease" (Fallot tetralogy). There were no other obsessions, and no compulsive behaviors were noticed.

Several months after these initial symptoms, the patient started having incomprehensible fears, refused to be left alone, locked all doors and windows, and did not go outside in an overwhelming fear that someone would harm her.

Over the next years the frontotemporal dementia picture developed. The patient's verbal output started to vanish until "she created a kind of a new dialect, [and the family] could only understand some muttered swear-words"; she was sexually uninhibited, impulsive, and aggressive even with her grandchild; and she started to eat without rules, even mayonnaise with butter. She had complete lack of insight to these changes.

Upon examination she was coprolalic, inadequate, and perseverant, and all frontal release signs could be easily elicited. A brain MRI showed left-predominant bilateral frontotemporal atrophy, the same region having significant hypoperfusion on SPECT scan. Comprehensive neuropsychological testing was compromised by the striking language dysfunction.

\section{Discussion}

Frontotemporal dementia has been regarded as a clinically and anatomically heterogeneous disorder, and by this way traditionally subdivided in frontal and temporal subtypes by the predominance of behavioral features in the former and of language or semantic knowledge in the latter. ${ }^{1}$ Our patient fulfils the proposed criteria for the behavioral variant of frontotemporal dementia. In this, many neuropsychiatric symptoms have been described; the most common are included on the clinical diagnostic criteria for frontotemporal dementia. ${ }^{4}$

Hypochondriacal symptoms can on occasion be found in frontotem- poral dementia, ${ }^{3}$ but they are highly unusual in the first stages of disease and have not been, to our knowledge, reported as the presenting sign. Nevertheless, they preceded by years the diagnosis of Huntington's disease, ${ }^{5}$ and the disruption of fronto-subcortical circuitry seems to be the most reasonable explanation, providing an attractive anatomical background for what we have seen in our patient. Because true hypochondriasis in our patient could not ever be a true diagnostic consideration, the reported case highlights the need to consider this masked diagnosis in all patients presenting with novel and atypical psychiatric symptoms in late life.

Álvaro Machado, M.D.

Neurology Department, Hospital de São Marcos, Braga, Portugal

Sónia Simões, M.D.

Psychiatry and Mental Health

Department, Hospital de São

Marcos, Braga, Portugal

\section{References}

1. Snowden JS, Neary D, Mann DMA: Frontotemporal Lobar Degeneration: Frontotemporal Dementia, Progressive Aphasia, Semantic Dementia. New York, Churchill Livingstone, 1996

2. Mendez MF, Perryman KM: Neuropsychiatric features of frontotemporal dementia: evaluation of consensus criteria and review. J Neuropsychiatry Clin

Neurosci 2002; 14:424-429

3. Gregory CA: Frontal variant of frontotemporal dementia: a cross-sectional and longitudinal study of neuropsychiatric features. Psychol Med 1999; 29:1205-1217

4. Neary D, Snowden JS, Gustafson L, et al: Frontotemporal lobar degeneration: a consensus on clinical diagnostic criteria. Neurology 1998; 51:1546-1554

5. Arrojo Romero M, Silva AF, Palha AP: Hypochondriasis and Huntington's disease. Actas Esp Psiquiatr 2006; 34:65-66 